\title{
Papel de los aleloquímicos en el manejo de los recursos naturales
}

\author{
ANA LUISA ANAYA LANG ${ }^{1}$
}

\begin{abstract}
RESUMEN. El presente trabajo se refiere a los aspectos más sobresalientes de la investigación sobre los compuestos secundarios de las plantas y a su aplicación en beneficio del manejo de los recursos naturales. En relación con ésto, se enfatiza la importancia del estudio de los agroecosistemas tradicionales, con el fin de obtener información sobre el manejo de recursos tanto en el espacio como en el tiempo algunas veces los campesinos utilizan de manera empírica el potencial fitoquímico algunas plantas para controlar malezas o insectos nocivos. De estas ( especialmente plantas cultivadas y silvestres) investigaciones podremos obtener los datos necesarios que nos permitan descubrir recursos potenciales de utilidad en el manejo.
\end{abstract}

ABSTRACT. In this paper some aspects of research on secondary compounds of plants are covered, in particular those which deal with their applications for the management of traditional agricultural systems. Traditional agriculturalists often make empiric use of the phytochemical potentiality of plants. The recover of this information might be of great practical importance.

\section{INTRODUCCION}

Por recursos naturales no solo entendemos a aquellos elementos de la naturaleza que se incorporan directamente al proceso productivo, sino al conjunto de ecosistemas que constituyen el entorno del hombre, es decir plantas, animales, microorganismos, agua, suelo, aire, luz, guardando una estrecha interacción entre sí, y un equilibrio dinámico con la suma total de la vida sobre la Tierra.

Cualquier actividad que esté dirigida a la obtención de un beneficio directo o indirecto a partir de algún recurso natural, sea éste renovable o no, y que implique un cambio en la dinámica natural del recurso, una desviación de su curso normal, un cambio de lugar, el fomento de su cantidad, el cuidado y estímulo de su crecimiento, en pocas palabras, su dirección, su control o su manipulación, puede traducirse como manejo.

${ }^{1}$ Instituto de Fisiología Celular, Universidad Nacional Autónoma de México., Apdo. Postal 70-600, Delegación Coyoacán 04510, México, D. F. 
Las formas mas antiguas de manejo de recursos pueden ejemplificarse con la aparición de la agricultura y la irrigación.

La agricultura es una de las actividades mas importantes que el hombre ha realizado; de ella dependen la obtención de la mayor parte de sus alimentos y de muchas materias primas utilizadas para cubrir diversas necesidades de vivienda, vestido, medicinas, industria, recreación, etc. La agricultura abarca también a la silvicultura, la horticultura, la pesca, la jardinería y la ganadería como formas principales de manejo de recursos terrestres y acuáticos.

$\mathrm{El}$ avance tecnológico de la agricultura en el presente siglo, ha logrado aumentos impresionantes en la producción de alimentos a nivel mundial, mismos que no se hubieran logrado sin este avance tecnológico (Stoll, 1986); pero el precio que se ha pagado por ésto ha sido muy alto, desde el punto de vista ecologico y social. Por ello cabe preguntarnos si las semillas mejoradas, los tractores, la homogenización de las parcelas, el mayor tonelaje por hectárea, las enormes superficies de monocultivos en búsqueda de la producción masiva de alimentos para el ganado, el uso indiscriminado de pesticidas, fertilizantes y otros químicos, serán la respuesta a la creciente demanda de alimentos, serán la mejor opción de desarrollo agrícola para nuestro país, serán la solución para detener el deterioro del ambiente y la pérdida de recursos naturales como el agua, el suelo, la flora y la fauna. La crisis ecológica y agrícola que hoy enfrentamos puede llevarnos, si no la resolvemos adecuadamente, a la pérdida irreversible de muchos recursos naturales, indispensables para sobrevivir en el futuro.

La agricultura moderna convencional tiene serias desventajas entre las que podemos mencionar las siguientes:

1. pérdida de la variedad en la producción y por lo tanto, pérdida de la capacidad de autosubsistencia.

2. pérdida de la variedad genética de los cultivos.

3. desaparición de recursos bióticos silvestres, que se eliminan para no interferir o estorbar a los cultivos.

4. homogenización del agroecosistema y por lo tanto desequilibrio ecológico.

5. mayor peligro de erosión por el uso de maquinaria, falta de cubierta vegetal en el suelo y escasez de fuentes de materia orgánica para enriquecer continuamente al mismo.

6. necesidad de gran cantidad de insumos y por lo tanto de inversión.

7. contaminación por el uso indiscriminado de pesticidas, herbicidas, fertilizantes, etc.

Por otro lado, la agricultura tradicional tiene diversas características que la colocan, desde el punto de vista ecológico, por encima de la "moderna". Algunas de estas características son:

1. diversidad en la producción en el tiempo y el espacio.

2. producción intensiva.

3. conservación de la variedad genética de los cultivos.

4. conservación, manejo y uso de recursos silvestres paralelos al cultivo. 


\section{5. mayor equilibrio ecológico. \\ 6. mayor control de la erosión. \\ 7. fuentes abundantes de materia orgánica. \\ 8. consumo mínimo de productos industriales y por lo tanto menor contaminación. \\ 9. menor inversión.}

10. mayores posibilidades de autosubsistencia.

11. mayor necesidad de mano de obra.

12. conservación y evolución creciente del conocimiento y por lo tanto de la cultura.

No se trata con esta comparación, de calificar a la tecnología como el enemigo irreconciliable de la naturaleza y de la agricultura. No se trata de volver al primitivismo en las formas de producción. Se trata de "modernizar" a la agricultura de modo que el criterio ecológico y una visión integral de manejo de recursos, con base en las necesidades de la población y en la cultura que posee, sean los que marquen las pautas para el uso del suelo y para la producción.

En los sistemas agrícolas manejados ecológicamente, la productividad por unidad de área de un cultivo puede reducirse, pero otras características ambientales, como la capacidad de uso múltiple del habitat, se ven favorecidas. El resultado neto de ésto, se traduce en una gran diversidad de recursos y una estabilidad ecológica general. Respecto a ésto, puede argumentarse que éstas no son ventajas suficientemente sustanciales y tangibles desde el parcial punto de vista de la producción, sin embargo la creciente escasez de energéticos y la inflación irrefrenable, probablemente demostrarán que los aspectos financieros a corto plazo ya no serán más la principal prioridad en agricultura. Posiblemente la conservación de energía y la calidad ambiental asumirán dicho papel en un futuro cercano.

Nos enfrentamos al tratar de resolver este problema, con asuntos de órden biológico, geográfico, ecológico, climático, social y económico, y además matizando con mayor fuerza cada vez a los anteriores, con una cuestión ética de primordial importancia: la actitud humana frente a la naturaleza, su forma de explotación y el reto enorme que significa enderezar el camino y resarcir los errores que han provocado el deterioro de la biosfera entera.

El objetivo principal de éste trabajo, se refiere a una de las formas de contribución del biólogo a la solución de los graves problemas que el mundo en general y el país en particular enfrentan para producir mas y más alimentos, y más y más recursos que demandan, por un lado, una población que crece explosivamente y por el otro, un complejo sistema industrial y comercial, que requiere toda clase de insumos provenientes de los recursos naturales. Por ello es necesario multiplicarlos para que puedan satisfacer estas demandas, y buscar además nuevas fuentes de recursos, más apropiados o que sustituyan a los que se han agotado.

Como el problema a todas luces es muy complejo, debemos esperar que las soluciones también lo sean, sin embargo, estas soluciones se estan ya generando a través de la investigación científica. La biología y la ecología de los agroecosistemas deben ser temas constantes de estudio para generar soluciones, instrumentos que nos permitan encontrar caminos mas racionales de producción, sin destruir, sin propiciar la extinción, sin contaminar, enriqueciendo el germoplasma y la cultura, mejorando la producción 
con el uso de recursos múltiples, descubriendo el uso potencial de muchos organismos y aprovechando optimamente el espacio, el agua y el suelo, para producir intensivamente y no extensivamente.

\section{EL PAPEL DE LOS COMPUESTOS SECUNDARIOS EN EL MANEJO DE LOS RECURSOS BIOTICOS.}

Las plantas superiores poseen como resultado de los diversos procesos evolutivos, un número inmenso y variadísimo de compuestos químicos secundarios. Los compuestos o metabolitos secundarios se llaman así, porque no se encuentran presentes de manera general en toda la materia viva; por ésto contrastan con los compuestos primarios (proteínas, lípidos, carbohidratos y ácidos nucléicos) que siempre estan presentes.

Los compuestos secundarios derivan de un grupo reducido de compuestos precursores: ácido acético y algunos aminóacidos esenciales (fenil alanina, tirosina y triptofano). A pesar de esta reducida fuente de orígen, el número y variedad de compuestos secundarios, como se mencionó, es enorme. Desde el punto de vista biosintético, los metabolitos secundarios se dividen en:

- fenil propanos

- acetogeninas

- terpenoides

- esteroides

- alcaloides

- y un grupo diverso de compuestos que no encajan dentro de estos cinco grupos (Whittaker y Feeny, 1971).

Acidos orgánicos, fenoles, cumarinas, glucósidos, terpenos, quinonas, hormonas, saponinas, alcaloides, tioles, etc., metabolitos secundarios simples y complejos, aromáticos y de cadena abierta, se van multiplicando en los diversos taxa, tanto de plantas, animales como microorganismos, y a la vez desempeñando diferentes funciones biológicas y ecológicas.

En relación con la función que desempeñan los metabolitos secundarios, podemos afirmar que ésta es múltiple, segun actúen en las diferentes esferas o niveles de la biología o el nicho ecológico de un organismo. Su función metábolica es aun muy oscura, y en cambio su función dentro de las comunidades, como instrumentos de comunicación entre los diversos organismos, en muchos casos es bien conocida. La ciencia que estudia el papel que los metabolitos secundarios desempeñan dentro de la comunidad y dentro de una población, es la ecología química. Podemos hablar de ecofisiología química, cuando se trata de investigar el papel que los metabolitos secundarios desempeñan dentro de un organismo y dentro de una célula. Ahora bien, la ecología química abarca en su campo de estudio, otro tipo de fenómenos naturales como son: los ciclos biogeoquímicos y la contaminación.

Los metabolitos secundarios que median interacciones entre organismos de diferente especie, reciben el nombre de aleloquímicos. Estos se pueden dividir a su vez en: a) alomonas, que son aquellos metabolitos secundarios que le dan ventajas al 
organismo que los produce; b) kairomonas, que le confieren ventajas al organismo que recibe la acción del metabolito secundario; y c) depresores, que sin darle ventajas al organismo productor, perjudican al receptor.

Los metabolitos secundarios que median interacciones entre organismos de la misma especie, se dividen en tres grupos según su efecto:

a) autotoxinas; b) autoinhibidores adaptativos; y, c) feromonas (Whittaker y Feeny 1971).

Si los metabolitos secundarios resultan de gran importancia para las diversas interacciones biológicas que se establecen entre los organismos de una comunidad, resulta obvio que podemos obtener ventajas de su manejo directo o indirecto, con el fin de optimizar la producción dentro de un agroecosistema. Llamar la atención sobre la importancia de ésto, es una de las intenciones de este trabajo.

\section{LA ALELOPATIA COMO MECANISMO DE CONTROL NATURAL DE ARVENSES EN LOS CULTIVOS.}

La alelopatía es un fenómeno que puede definirse como el efecto inhibitorio o estimulante de diversos metabolitos secundarios liberados al medio por un organismo (generalmente una planta), sobre el crecimiento, metabolismo y desarrollo de otros organismos. En este proceso pueden estar involucrados también los animales y los microorganismos.

La alelopatía como proceso ecológico, actúa de manera simultánea y/o sinergística con otros procesos como la competencia. Cuando la alelopatía y la competencia actúan conjuntamente, se habla entonces de interferencia, y en este caso es sumamente difícil diferenciar a ambos efectos en el medio natural. En la mayoría de las interacciones entre cultivo y arvenses, ambos efectos aparecen involucrados, lo que dificulta el estudio de los mismos.

Los compuestos alelopáticos pueden ser liberados al medio por diversos caminos:

1. volatilización de las partes aereas de una planta, las hojas principalmente.

2. exudación a través de las raíces.

3. lixiviación o lavado de las partes aereas de las plantas por la lluvia.

4. descomposición de la materia orgánica muerta.

\section{PRODUCTOS NATURALES IDENTIFICADOS COMO ALELOPATICOS}

Los compuestos químicos secundarios que estan implicados en la alelopatía, petenecen a muy diversos grupos de sustancias. Según Putnam (1985), se pueden dividir arbitrariamente en los siguientes grupos:

Gases tóxicos, ácidos orgánicos, aldehídos, lactonas simples insaturadas, cumarinas, quinonas, flavonoides, taninos, alcaloides, terpenos, esteroides, y finalmente, un grupo misceláneo de compuestos que incluyen ácidos de cadena larga, alcoholes, polipéptidos 


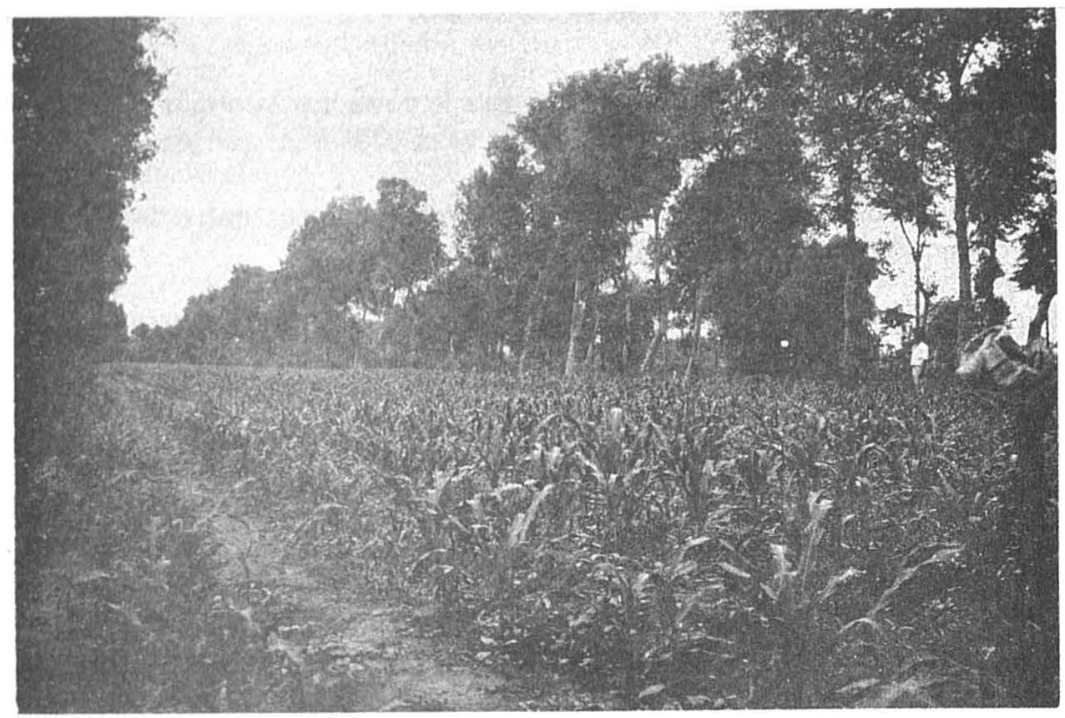

Figura 1. Los "camellones" de Tlaxcala, representan uno de los agroecosistemas tradicionales donde el manejo de los recursos, tanto las plantas cultivadas como las silvestres, el suelo, el agua, los abonos naturales, la combinación y la rotación de cultivos, se hace de una manera integral.

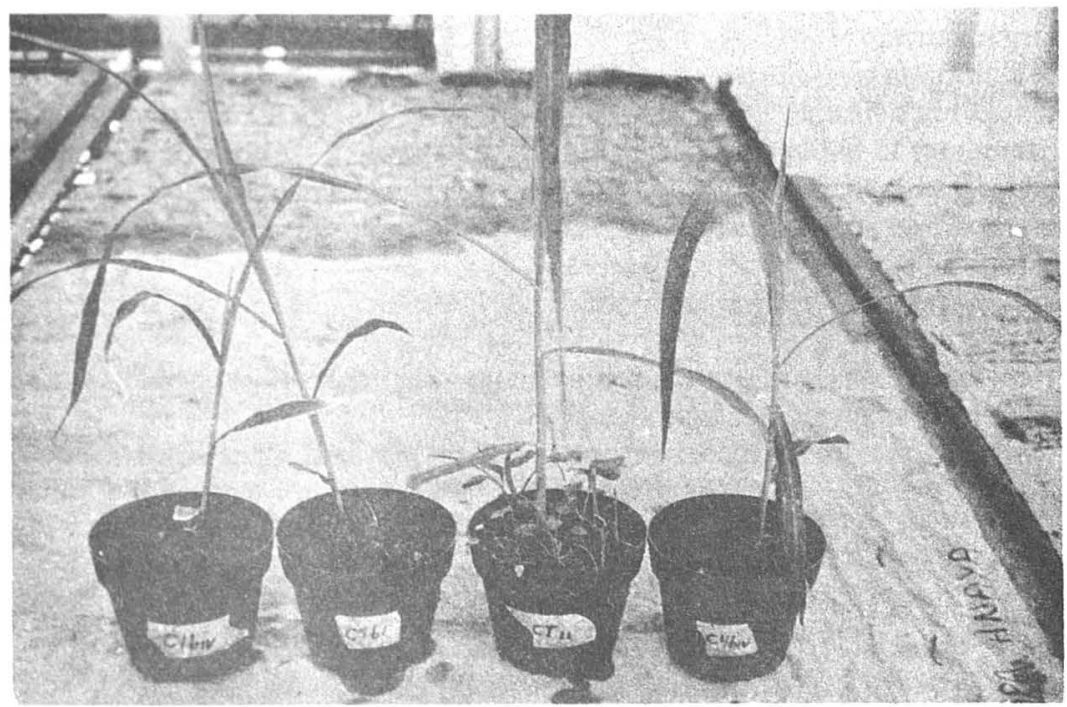

Figura 2. Es necesario realizar una amplia investigación, sobre la posibilidad de usar diversas plantas эrvenses con potencial alelopático, como abonos verdes que mezclados con el suelo, disminuyan la presencia le otras malezas en los cultivos. Las tres macetas con menor crecimiento de malezas tienen mezcladas en el suelo, tres especies de arvenses comúnes, con potencial alelopático. 
y nucléosidos. Se supone que muchos alelopáticos son compuestos secundarios de las plantas, que tienen un papel protector para las mismas, incluídas diversas resistencias al ataque de insectos y microorganismos. Swain (1977) reportó que mas de 10,000 metabolitos secundarios de bajo peso molecular, han sido aislados de las plantas verdes y de los hongos, y calculó que el número total de compuestos aislados e identificados es de alrededor de 400,000. Estas sustancias y sus análogos químicos, constituyen una fuente valiosa para la síntesis de nuevos herbicidas en el futuro, especialmente para el control de malezas por medio de sustancias naturales. El mejoramiento de cultivos (quizás a través de la ingeniería genética) puede asimismo producir plantas que eliminen a sus competidores o que sirvan de fuentes naturales de diversas sustancias químicas útiles.

\section{METODOS DE PROTECCION NATURAL DE CULTIVOS.}

Estos métodos han adquirido una gran importancia, pues ofrecen una respuesta sencilla y accesible para combatir malezas y plagas. La naturaleza nos ofrece una amplia variedad de plantas para usarlas como protección natural de los cultivos, un potencial que debe aprovecharse cabalmente y que lleva implícita una enorme diversidad de metabolitos secundarios.

La mayor parte de los campesinos en el Tercer Mundo, incluyendo a México, utilizan una serie de medidas directas e indirectas para salvaguardar los cultivos del ataque de enemigos naturales. La protección de los cultivos es sin embargo, un proceso complejo que requiere del entendimiento de las interacciones entre el medio y sus elementos, las prácticas agrícolas y el sistema predominante de cultivo, del clima y la región.

Debemos plantearnos la pregunta, respecto a que si la función del manejo de los recursos en los agroecosistemas es asegurar la máxima producción de los mismos. Esta meta ha sido trazada por la visión antropocéntrica que busca los máximos beneficios a corto plazo, sin considerar los costos ecológicos. Así que es necesario insistir, que es mucho mejor buscar un equilibrio óptimo entre las metas económicas y las ecológicas, que deje un espacio para otras formas de vida, otros recursos. Es evidente, sin embargo, que cuando las plagas y malezas ponen en peligro el alimento y la existencia humanas, no es fácil dejarlos de ver como enemigos, en esto radica la dificultad del reto que enfrentamos. Altieri (1983) afirma que la adopción de estrategias bio-ambientales en el manejo de los cultivos, incluye la estabilización de la producción, más que maximización de la misma; lo óptimo en este caso no es equivalente a lo máximo.

Los sistemas tradicionales de cultivo muestran un panorama de alta variedad biológica; en ellos crecen simultáneamente, compartiendo ordenadamente el espacio, diversos cultivos y plantas silvestres(Fig. 3). Esta heterogeneidad constituye por sí misma una forma de protección preventiva que surge de manera natural del manejo agrícola múltiple, debido entre otras, a las siguientes razones:

1. hay una gran disponibilidad de habitats pequeños y una multiplicidad de fuentes de alimentos. 
2. los movimientos y la conducta migratoria de las plagas, están influidas por la atracción visual y aromática de las plantas hospederas y no hospederas, así como por los microclimas; de esta manera pueden confundirse y desorientarse, y por lo tanto se muestran incapaces de detectar con facilidad a la planta que les sirve de alimento.

3. la cobertura del suelo debido a la combinación de plantas cultivadas es más amplia, a veces es total durante todo el ciclo de cultivos, y solo la sombra producida por esta razón, elimina a aquellas especies de arvenses demandadoras de luz, lo que disminuye la necesidad de control de malezas.

4. los efectos repelentes e inhibitorios de algunas plantas en este tipo de agroecosistemas también reducen la dispersión de plagas o el crecimiento de malezas (Altieri $e t$ al., 1983).

Se citan a continuación algunos ejemplos de experiencias al respecto:

a) Los cafetales a la sombra en México, son agroecosistemas tradicionales que tienen una gran semejanza con los bosques caducifólios, debido a la gran diversidad de plantas que en ellos crecen, y a los tres estratos de vegetación que los forman: el estrato herbaceo, el arbustivo (representado por el café) y el arbóreo (constituido por los árboles que sombrean al cultivo). Esto constituye una protección para el cultivo del café por la estabilidad ecológica que le brinda. Las interacciones biológicas son más variadas en ellos, lo que permite una gama amplia de alternativas de control natural sobre muchas plagas y malezas. El suelo que se encuentra en contacto inmediato con las plantas de café contiene una serie de compuestos inhibitorios que pueden extraerse con agua. $\mathrm{Al}$ probar el efecto de estos extractos sobre el crecimiento de diversas malezas, se encontró que produjeron una inhibición significativa del mismo (Anaya et al., 1982). Posteriormente, Waller et al., (1982), encontraron cantidades apreciables de cafeína y otros alcaloides en estos suelos, que se acumulan en forma creciente, a medida que el cafetal tiene mayor edad. Se pudo comprobar también, que el estrato herbaceo en este tipo de cafetales, está densamente poblado y casi exclusivamente por plantas de la familia comelinacea; al detectarse el potencial alelopático de las principales plantas de este agroecosistema, se pudo comprobar que los mayores efectos inhibitorios sobre el crecimiento de malezas lo produjeron precisamente los lixiviados de las comelinas. Las plantas vivas, las hojas secas y el liter de estas plantas produjeron una fuerte inhibición sobre Bidens pilosa. Los cafeticultores conocen este efecto y por ello promueven el crecimiento de las comelinas en el piso de los cafetales,(Fig. 4) o bien las "chapean" y las dejan que se descompongan en el suelo con el fin de abonarlo e impedir la invasión de otras especies. Este hecho comprueba que la alelopatía es un factor importante en la dinámica de las comunidades y que puede utilizarse para controlar la presencia de ciertas plantas "indeseables" en el agroecosistema (Ramos et al., 1983).

b) Los cultivos de maíz interplantados con calabaza, generalmente tienen una menor invasión de malezas, por el efecto de sombra de la calabaza en el habitat y la acción alelopática que esta planta ejerce sobre muchas malezas (Jiménezy Schultz, 1980, Chacon y Gliessman, 1982; Anaya et al., 1987.) Esto dá por resultado que la producción de maíz se vea menos afectada por las malezas, cuando el maíz se siembra en 


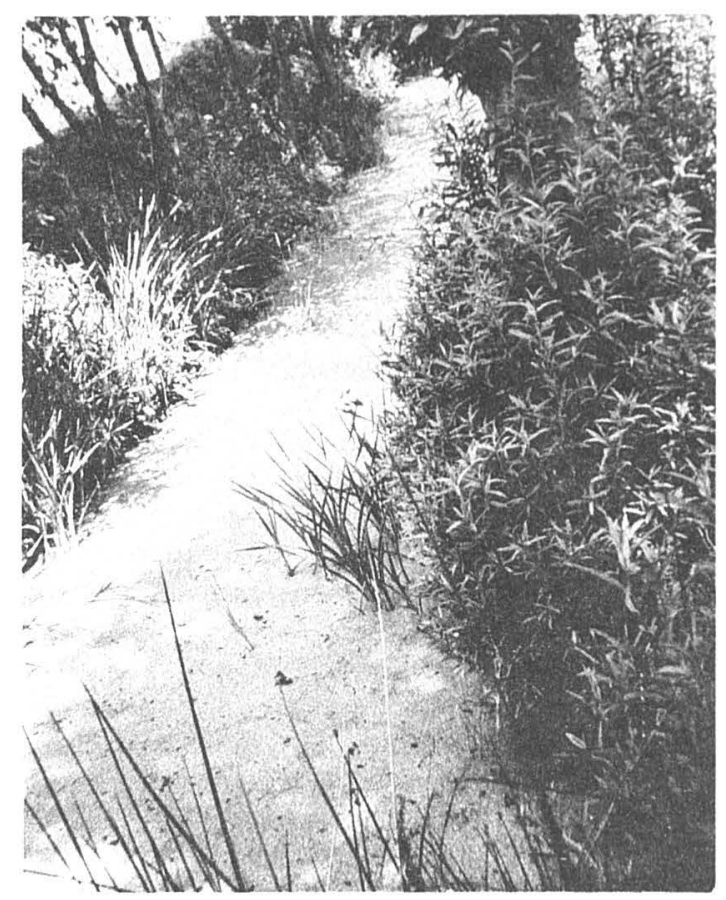

Figura 3. En los sistemas tradicionales de cultivo, no solo se realiza un racional y variado manejo de recursos, sino que paralelamente, se practica la conservación de recursos silvestres dentro de pequeñas zonas naturales de reserva que complementan los bancos de germoplasma de especies cultivadas con la riqueza genética vegetal y animal que poseen. En esta fotografía se observa un canal de agua que rodea a un "camellón".

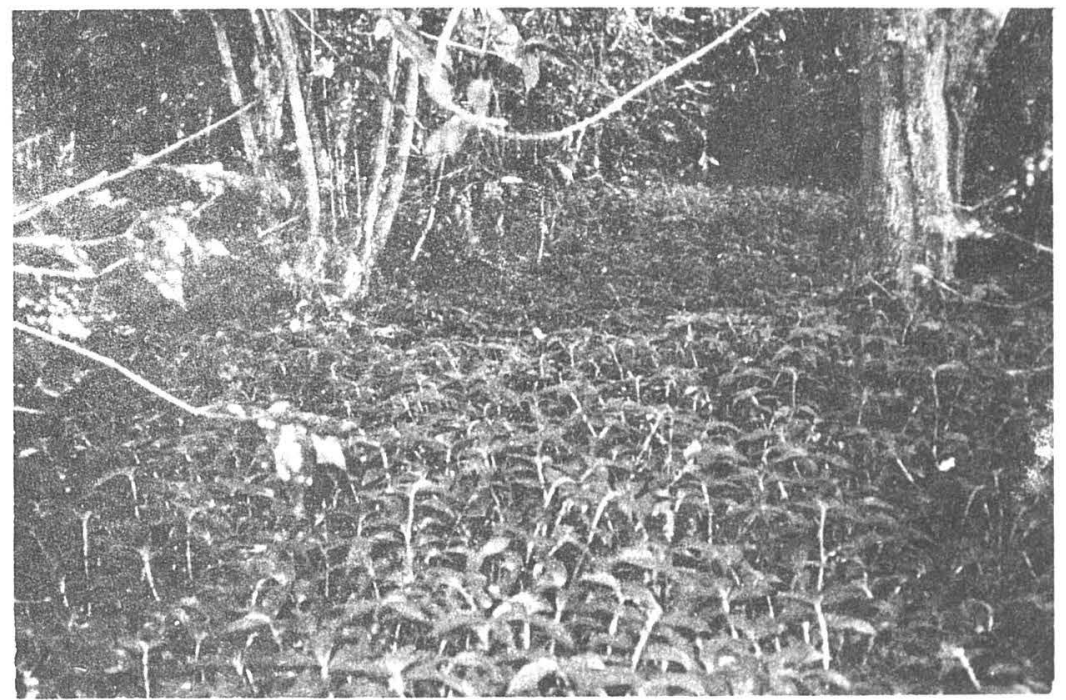

Figura 4. En los cafetales a la sombra en Coatepec, Veracruz, los campesinos utilizan como cultivos de cobertura a diversas especies de la familia de las Comelinaceas, que con su presencia y gracias al potencial alelopático que poseen, inhiben e! crecimiento de cualquier otra maleza dentro del cafetal. 
combinación con la calabaza o con el chilacayote. Jiménez et al, (1980) demostraron asímismo que el polen del maíz tiene un fuerte potencial alelopático, que parece impedir en la etapa en que más se produce, la fructificación de la misma calabaza, según lo señalan algunos campesinos. Posteriormente, Cruz et al., (1987), pudieron comprobar el efecto perjudicial de los extractos de polen de maíz sobre la respiración y la división celular de las células meristemáticas de la sandía.

c) El lirio acuático es usado como abono verde en los sistemas chinamperos; extendido sobre las parcelas y cubierto con lodo del fondo de los canales, esta planta considerada como una de las peores malezas acuáticas, sirve de fértil cama para que numerosos cultivos se desarrollen en ella. En un experimento realizado en Xochimilco, Anaya et al., (1987) encontraron que incrementa significativamente la producción de los cultivos, comparado con el efecto de los fertilizantes inorgánicos e incluso con el del estiercol de vaca. El lirio tiene además otro papel importante pues protege al suelo contra el exceso de evaporación superficial, impidiendo con ésto el depósito de sales y la intoxicación que éstas provocan a las plantas. En relación con el número total de malezas que aparecieron en las parcelas, se pudo notar una disminución significativa de las mismas en aquellas fertilizadas con lirio acuático.

d) Otro ejemplo sobre el efecto de una cubierta vegetal en el crecimiento de cultivos y malezas, lo tenemos en un experimento de campo llevado a cabo en Tlaxcala, donde se probaron dos cubiertas vegetales: hojas de Alnus firmifolia y Berula erecta conJuncus sp. El número total de malezas en las parcelas con cubierta vegetal, fué significativamente menor en comparación con las parcelas sin cubierta.(Fig. 5 y 6) De manera contraria, las producciones de frijol y calabaza fueron estimuladas por las cubiertas vegetales, al igual que el número de nódulos en las raíces del frijol (Anaya et al., 1987).

e) Chenopodium album es una arvense muy común que puede exudar cantidades tóxicas de ácido oxálico en la época en que está en floración e inhibir con ello la germinación y el crecimiento de otras plantas y organismos diversos (Caussanel y Kunesch, 1979). Bye (1979), comenta que los tarahumaras cultivan esta arvense combinándola en rotación con el maíz, para tener menor incidencia de malezas en sus milpas. Esto sugiere que las secreciones y residuos de esta planta tienen un efecto alelopático que se manifiesta, inhibiendo el crecimiento de varias plantas en sus cultivos.

Estos resultados abren un vasto campo de investigación multidisciplinaria, cuyos diversos objetivos deben enfocarse a la solución de las interrogantes sobre el papel de los metabolitos secundarios dentro de las comunidades agrícolas.

La combinación de diversos cultivos y variedades, puede retardar el inicio de una enfermedad, reduciendo la diseminación de esporas o modificando las condiciones microambientales como la humedad, la luz, la temperatura y los movimientos del aire (Browning y Frey, 1969; Larios, 1976). Ciertas asociaciones de plantas pueden funcionar como repelentes, interruptoras del crecimiento o tóxicas, sobre diversos microorganismos. En el caso de los patógenos del suelo, algunas combinaciones de plantas y abonos orgánicos pueden favorecer la fungistasis y antibiosis en los suelos (Sumner et al., 1981). 


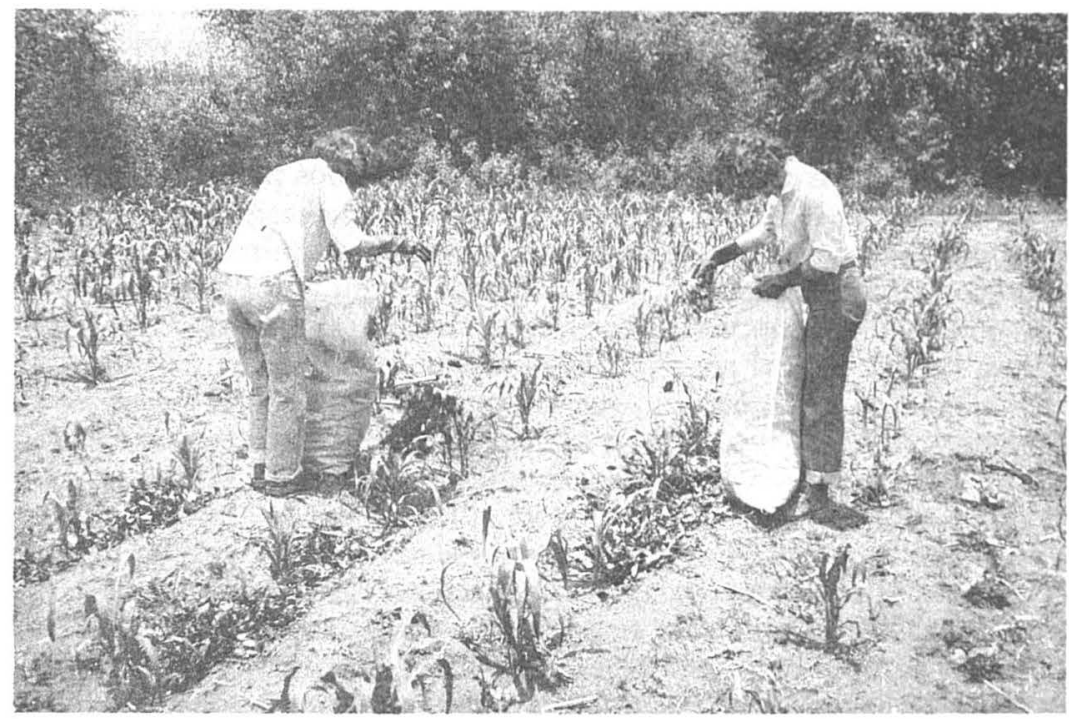

Figura 5. Algunas plantas silvestres pueden ser fuente de metabolitos secundarios con acción herbicida. Estas plantas pueden usarse como materia prima en la industria o bien aplicarse al suelo directamente durante el ciclo de cultivo, con objeto de controlar la población de arvenses.

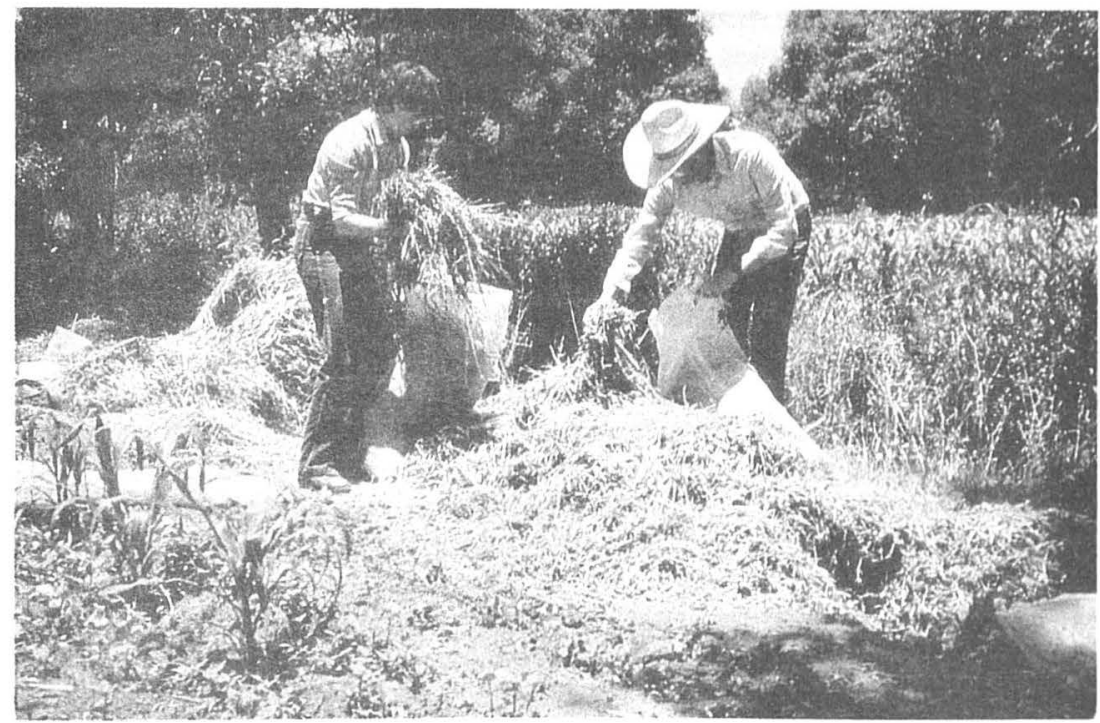

Figura 6. Las plantas verdes son la fuente más rica y diversa de metabolitos secundarios susceptibles de aprovecharse como insecticidas, fungicidas, antibióticos, etc. Solo a través de la investigación científica ampliamente reconocida y apoyada, se podrá obtener la información necesaria para aprovechar este potencial de las plantas. 
El entendimiento de las interacciones entre las plantas, los microorganismos, los insectos herbívoros y sus enemigos naturales, puede darnos pautas para mejorar los sistemas de control biológico. Las plantas pueden tener diversos efectos directos o indirectos, positivos o negativos, no solo sobre los herbívoros, sino también sobre otras plantas, insectos, microorganismos, y sus enemigos naturales. Por ejemplo, diversos caracteres químicos y físicos son esenciales para la localización de los hospederos entomófagos; las avispas del género Trichogramma, parasitan en mayor grado a los huevos de Heliothis sp. y Plusiinae sp., que son mariposas que atacan a diversos cultivos, cuando se encuentran sobre plantas de tomate, que cuando crecen sobre maíz, sorgo, tabaco o trébol. Seguramente algún caracter químico del tomate, atrae a las avispas de tal forma que éstas aumentan sus posturas sobre los huevos de estas plagas. Con base en ésto, pueden utilizarse plantas diversas y compuestos volátiles de las mismas, para mejorar o hacer más atractivo el habitat y la selección de los hospederos de los insectos parásitos de plagas. Recientemente se encontró que las avispas del género Trichogramma aumentan su ataque, parasitando a los huevos del gusano del maíz, cuando se esparcen sobre los cultivos extractos de Amaranthus sp. (Altieri, 1983).

Las diversas variedades de plantas cultivadas, poseen diferentes sustancias químicas secundarias y en cantidad variable dentro de sus tejidos. Sus defensas potenciales contra el ataque de los herbívoros, depositadas en estas sustancias, varía por esta razón, y de igual manera su potencial alelopático. Las sustancias secundarias como el gosipol en el algodón y los alcaloides en los lupinos, desempeñan un papel importante en la resistencia a las enfermedades y plagas. El mejoramiento genético de los cultivos, que ha puesto mucho énfasis en buscar plantas que tengan mayor rendimiento, ha eliminado muchas propiedades químicas de las mismas que les conferían ventajas en el sentido de la defensa y la competencia contra sus enemigos. Actualmente se intenta, por medio de la ingeniería genética, mejorar e introducir a otras variedades, diversas defensas químicas naturales que muchas plantas poseen.

\section{PRINCIPALES ASPECTOS QUE LA ALELOPATÍA DEBE INVESTIGAR DENTRO DE LOS AGROECOSISTEMAS}

1. Detección y conocimiento de todas las plantas, cultivadas y silvestres con potencial alelopático, de todos los factores que determinan cambios en la composición química de las plantas, de la etapa del ciclo de vida de las plantas en la que éstas contienen mayor cantidad de metabolitos secundarios y de los organismos de la comunidad que están involucrados en los procesos alelopáticos.

2. Creación de sistemas de pruebas biológicas apropiadas, para determinar el modo de acción de los metabolitos y la clase de organismos a los que de alguna manera afectan. Esto implica su efecto sobre la membrana celular, la división celular, la respiración y la fotosíntesis entre otros procesos.

3. Diseño de experimentos que permitan determinar el umbral de la actividad biológica. 
4. Diseño de controles que impidan la variación en los experimentos.

5. Interpretación y transferencia de los resultados del laboratorio e invernadero a las situaciones en el campo.

6. Aplicación de los conocimientos para el óptimo manejo de los recursos bióticos en el espacio y el tiempo.

7. Utilización de asociaciones benéficas de plantas: cultivos mixtos y combinación de cultivos con arvenses.

8. Utilización de abonos verdes y coberturas vegetales.

9. Manejo de arvenses, con base en el conocimiento de la fisiología de la germinación y el crecimiento, con el fin de inhibirlas, estimularlas o controlarlas.

10. Separación, identificación y síntesis de metabolitos con posible aplicación.

11. Uso de diversos compuestos químicos y microorganismos para destruir, estimular y controlar las poblaciones de arvenses.

12. Control de la pérdida en la producción debida a las diversas toxinas liberadas y acumuladas en el suelo por las plantas cultivadas y arvenses, el liter, los desechos agrícolas, los microorganismos, etcétera.

13. Estímulo y protección de los microorganismos benéficos, por ejemplo los fijadores de nitrógeno.

Los agroecosistemas naturales deben tomarse como modelos para las estrategias de manejo de recursos en los agroecosistemas. Imitando la diversidad y la estructura de especies que hay en bosques y selvas, y manteniéndolas al máximo por medio del cultivo de varias clases de plantas, con diversos hábitos de crecimiento (árboles, arbustos, hierbas y epífitas), los agricultores de las zonas tropicales y de muchas semitropicales y templadas, pueden minimizar el riesgo de las plagas que abundan en condiciones de inestabilidad ecológica (Altieri, 1983). Capitalizando el conocimiento de las interacciones y asociaciones benéficas entre diversas plantas, a través de la investigación sobre los cultivos múltiples, la agricultura tradicional y la etnobotánica, puede lograrse la creación de sistemas que utilicen más eficientemente los recursos, mejoren la productividad general, amortigüen las epidemias y el deterioro ambiental, al mismo tiempo que conservan la riqueza biológica del ecosistema. Por otro lado este tipo de investigaciones abre automáticamente el camino a la búsqueda de recursos potenciales dentro de las comunidades, especialmente desde el punto de vista del hallazgo de compuestos químicos susceptibles de aprovechamiento. 


\section{LITERATURA CITADA}

ALTIERI, M.A. 1983. Agroecology. Berkeley, California.

ALTIERI, M.A., P.B. MARTIN y W.J. LEWIS 1983. A quest for ecologically based pest management systems. Environmental Management 7: 91-100

ANAYA, A.L., G. ROY-OCOTLA, L.M. ORTIZy L. RAMOS 1982. Potencial alelopático de las principales plantas de un cafetal. En: Jiménez Avila E. y A. Gómez Pompa, A. (Edrs.). Estudios Ecológicos en el Agroecosistema Cafetalero. CECSA, México. pp 15-92.

ANAYA, A.L., L. RAMOS, R. CRUZ y J. HERNANDEZ 1987. Allelopathy in Mexico. En: Waller, G.R. (Edr.). Allelochemicals: role in agriculture and forestry. ACS Symposium Series 330. Washington. pp 89-101.

ANAYA, A.L., L. RAMOS, R. CRUZ, J. HERNANDEZ y V. NAVA. 1987. Perspectives of studies on allelopathy in Mexican traditional agroecosystems: a case study in Tlaxcala. J. Chem. Ecol. 13(10): 2083-2101.

BYE, R.A. JR. 1979. Incipient domestication of mustards in Northwest Mexico. The Kiva 44 (2-3): 237-256.

BROWING, J.A. y K.J. FREY 1969. Multiline cultivars as a means of disease control. Ann. Rev. Phytopathol. 7: 355-382.

CAUSSANEL, J.P. y G. KUNESCH. 1979. Qualitative and quantitative study of growth inhibitors in root exudates of common lambsquarters (Chenopodium album L.) at the beginning of its flowering on hydroponic culture and under controlled conditions. Z. Pflanzenphysiol 93: 229.

CRUZ ORTEGA, R., A.L. ANAYA y L. RAMOS 1987. Effects of allelopathic compounds of corn pollen on respiration and cell division of watermelon. J. Chem. Ecol. 14(1): 71-86.

CHACON, J.C. y S.R. GLIESSMAN 1982. Use of the "non-weed" concept in traditional tropic agroecosystems of Southeastern Mexico. Agro-Ecosystems 8: 1-11.

JIMENEZ, O.J.J. y C. K. SCHULTZ 1981. Interacciones entre las plantas cultivadas y las plantas arvenses en una chinampa. Tesis. Fac. Ciencias, UNAM, México.

LARIOS, J.F. 1976. Epifitiología de algunas enfermedades foliares de la yuca en diferentes sistemas de cultivo. Tesis de Maestro en Ciencias. UCR/CATIE Turrialba, Costa Rica.

PUTNAMN, A.R. 1985. Weed allelopathy. pp. 131-155. En: Duke S.O. (Edr.) Weed Physiology. Vol. I. Reproduction and Ecophysiology. CRC Press, Inc. Florida.

RAMOS, L., A.L. ANAYA y J. NIETO DE PASCUAL. 1983. Evaluation of the allelopathic potential of the dominant herbaceous species in a coffee plantation. J. Chem. Ecol. 9(8): 1079-1097.

STOLL, G. 1986. Natural Crop Protection. Agrecol. Verlag Josef Margraf, Germany.

SUMNER, D.R., B. DOUPNIK, JR. y M.G. BOOSALIS. 1981. Effects of reduced tillage and multiple cropping on plant diseases. Ann. Rev. Phytopathol. 19: 167-187.

SWAIN, T. 1977. Secondary compounds as protective agents. Ann. Rev. Plant Physiol. 28: 479.

WALLER, G.R., D. KUMARI, J. FRIEDMAN, N. FRIEDMAN y CH. CHOU. 1986. Caffeine autotoxicity in Coffea arabica L.pp. 243-269. En: Putnam, A.L. y Ch. Tang (Edrs.). The Science of allelopathy. John Wiley \& Sons, New York.

WHITTAKER, R.H. y P.P. FEENY. 1971. Allelochemicals: Chemical interactions between species. Science 171: 757-770. 